\title{
Instability of the Mandelbrot Set
}

\author{
J. Peinke, J. Parisi, and B. Röhricht \\ Physikalisches Institut II, Universität Tübingen \\ O. E. Rössler \\ Institut für Physikalische und Theoretische Chemie, Universität Tübingen
}

Z. Naturforsch. 42 a, 263-266 (1987); received November 26, 1986

\begin{abstract}
Numerical evidence is presented that perturbations away from analyticity change the qualitative structure of the Mandelbrot set. The changes affect both the form and the surface structure. Most characteristically, the infinitely thin "necks" disappear. At at least one place, the surface structure becomes smooth.
\end{abstract}

The Mandelbrot set is found in a cut through the parameter space of the complex-analytic logistic map $\left(z_{n+1}=z_{n}^{2}+c\right)$ such that analyticity is preserved [1]. Such points in the $c_{\mathrm{re}}, c_{\mathrm{im}}$ plane for which an attractor exists for finite initial conditions are plotted white, the others black (or vice versa). There is a theorem [2] that it suffices to check the divergence or non-divergence, respectively, of a single initial condition - the origin. Specifically, in the case of non-divergence the corresponding Julia set (a chaotic repellor [3]) is connected, acting as a fractal basin boundary $[4,5]$. For the familiar picture of the Mandelbrot set, see Figure 1. Much more detailed and artful renderings may be found in $[1,5]$.

The Mandelbrot set is characterized by a rich, self-similar surface structure. A circular "bud" reappears on its own surface indefinitely often (see Figs. 1, 2a, 3a for three steps). For further details like filaments and seahorses, see [5]. The Mandelbrot set is easy to generate numerically as mentioned. To what extent do the properties of this set depend on the analyticity assumption made?

We investigated the following set of equations:

$$
\begin{aligned}
& x_{n+1}=x_{n}^{2}-y_{n}^{2}+a x_{n}+c_{\mathrm{re}}, \\
& y_{n+1}=2 x_{n} y_{n}+c_{\mathrm{im}} .
\end{aligned}
$$

For $a=0$ this is the complex-analytic logistic map mentioned above. Figure 2 shows a juxtaposition of one of the larger buds of the Mandelbrot set (see the arrow in Fig. 1) on the one hand, and the same

Reprint requests to Dr. J. Parisi, Physikalisches Institut II der Universität Tübingen, Auf der Morgenstelle, D-7400 Tübingen. region of parameter space, as far as the values of $c_{\mathrm{re}}$ and $c_{\text {im }}$ are concerned, but with a non-zero value of the third dimension $a$. Think of a tree trunk that has been cut in parallel to a particular exceptional plane (the Mandelbrot plane).

Figure 3 analogously shows a larger magnification of the boundary of the bud of the original Mandelbrot set (left), juxtaposed to the "corresponding" bud in a parallel cut, but ten times closer to the exceptional $(a=0)$ plane this time. Similar pictures can be obtained for higher magnifications and correspondingly smaller values of $a$. In each case, one finds a distortion of the former spherical shape; formation of a "neck region" of finite thickness and length; as well as changes of the surface structure. It should be stressed explicitly that smaller magnifications show a much stronger resemblance to the original Mandelbrot set, whereas stronger magnifications show progressively stronger deviations (see Figs. $2 b$ and $3 b$ ).

Now the main grain of salt: These pictures were obtained from the same initial condition, $x_{1}=y_{1}=0$, as the Mandelbrot set on the left itself. However, this particular initial condition ceases to play a mathematically privileged role outside the Mandelbrot plane as is well known [5]. We therefore in Fig. 3 also checked the influence of choosing randomly different initial conditions (ten on a circle of radius $0.1 a$ around the origin) and found no qualitative difference not only at the magnification shown, but also at a ten times higher magnification (in the region of the "dog's head" marked by an arrow in Figure $3 b$ ).

The same problem arises with the second main result to be reported: The formation of "bald" 


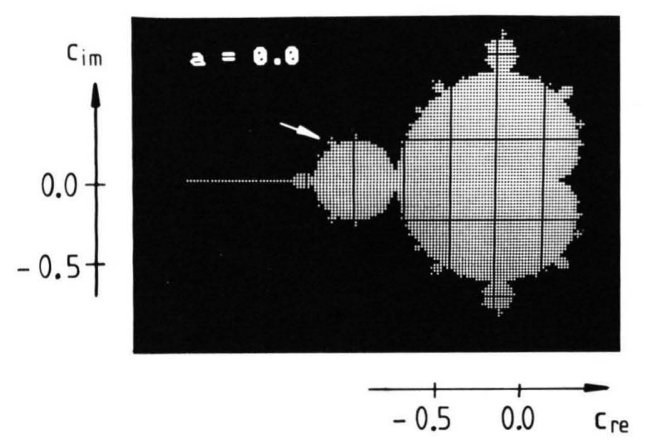

Fig. 1. The Mandelbrot set.
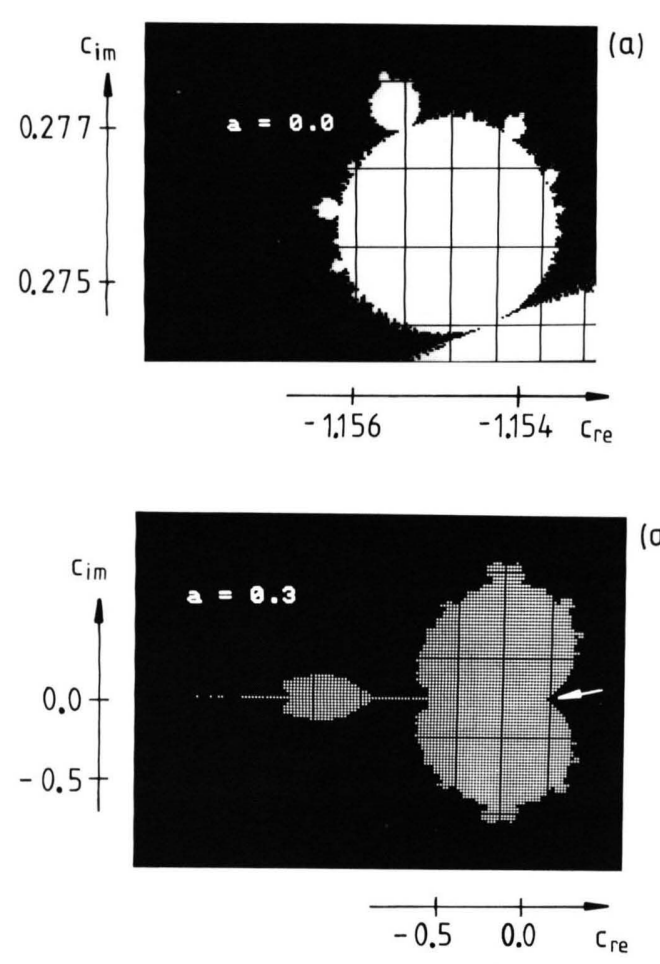
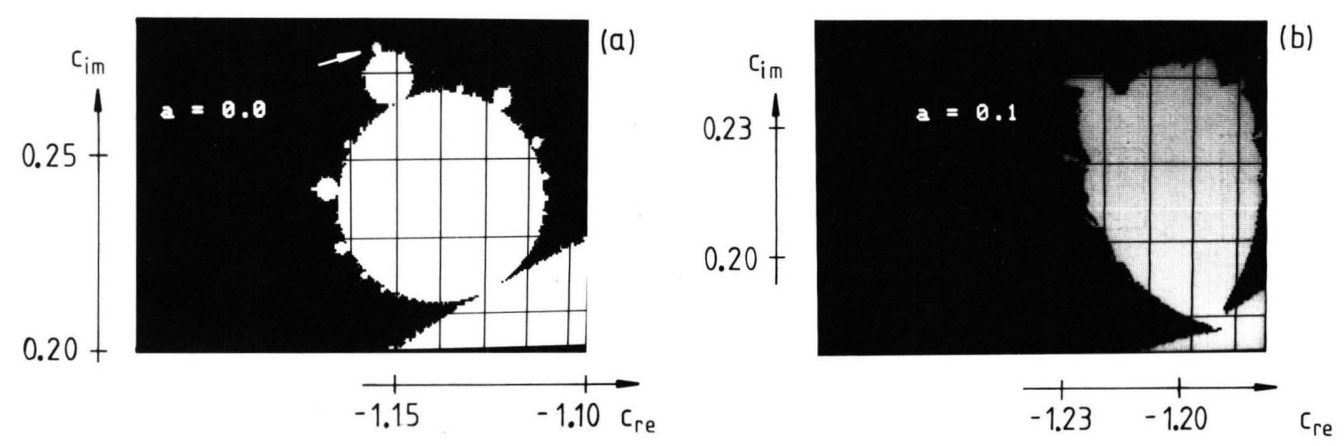

Fig. 2. (a) Blow-up of a region of the Mandelbrot set (marked in Fig. 1); (b) Corresponding region of a cut through parameter space parallel to the Mandelbrot set at $a=0.1$.

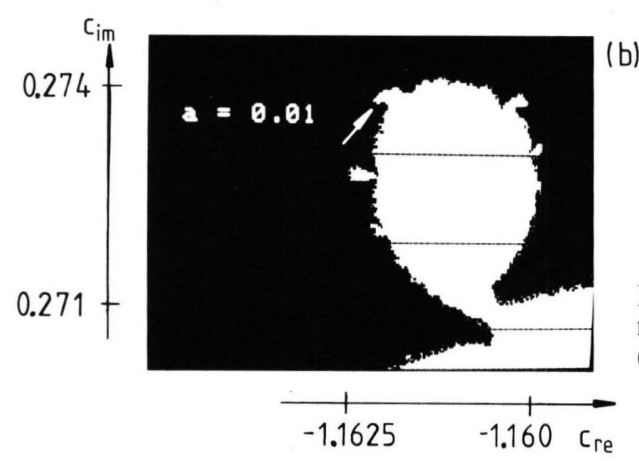

Fig. 3. (a) Blow-up of the region of the Mandelbrot set marked in Fig. $2 \mathrm{a}$; (b) Corresponding region for $a=0.01$ (compare Figure 2).
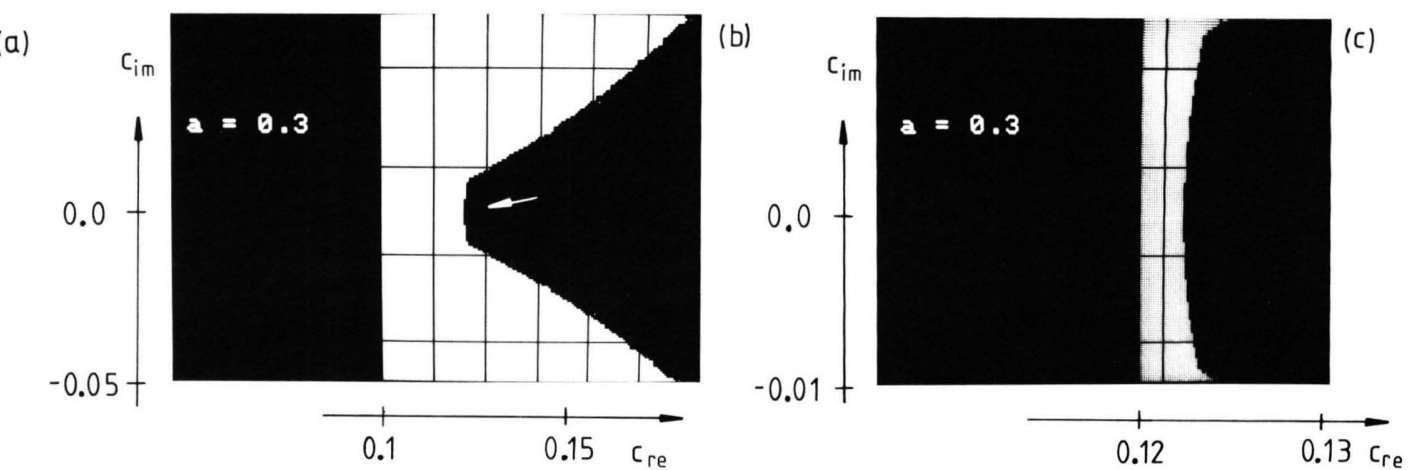

Fig. 4. (a) Distorted analogue to Mandelbrot's set at $a=0.3$; (b) Blow-up of the region shown in (a); (c) Blow-up of the region shown in (b). 


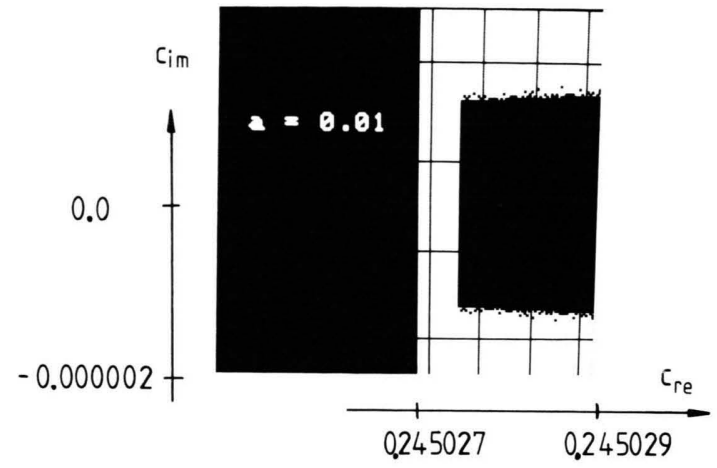

Fig. 5. High magnification of a picture analogous to Fig. $4 \mathrm{c}$, for $a=0.01$.

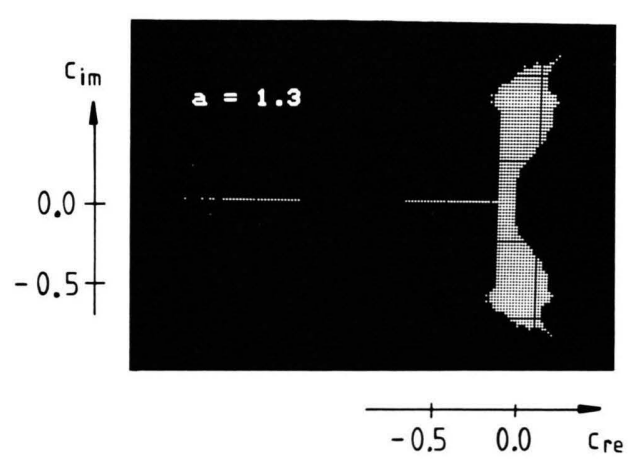

Fig. 6. "Remnant" of the Mandelbrot set at $a=1.3$.

stretches on the surface of the perturbed Mandelbrot set (Figure 4). The main indentation on the right of the Mandelbrot-set analogue of Fig. 4 a, on the $x$-axis, is shown in several magnifications in Figures $4 \mathrm{~b}$, c. The funnel has been "plugged". The surface of the plug appears curvilinear. This remains so at higher magnifications (up to a factor of $10^{4}$ ). All these pictures were obtained for many (about 20) different initial conditions (lying on two circles of radius $a / 3$ and $a / 30$, respectively). In all these runs, the same boundary points were found numerically. Figure 5 shows an analogous plug, at the same place but smaller, for a value of $a=0.01$ (that is, 30 times closer to the special plane $a=0$ ). Note the apparent collision of the curvilinear plug with a still fractal surface structure. The magnification this time is $10^{6}$; the initial condition is the origin.
Since the "baldness region" (width of the plug) is proportional empirically to $a^{\gamma}$ with an exponent of about 2.5 at five different tested values, we conjecture that the corresponding distortion of the Mandelbrot set arises already for arbitrarily small deviations from non-analyticity.

Our results suggest that there exists a "natural sequence" (universal?) of steps in the deterioration of the shape of the Mandelbrot set. So far, only a single dimension in parameter space $(a)$ was looked at, and only a limited numerical resolution was applied. Note, however, that bulk points (white in the above pictures) can be trusted numerically to the same extent as they can in the numerical computation of the Mandelbrot set proper. In either case computation times are the only means for a numerical check [6]. Other parameter dimensions are presently being investigated by Kahlert [7]. Moreover, the numerical resolution can also be drastically increased ([7] and work in preparation).

It seems that the Mandelbrot set "decays" in a lawful manner. Figure 6 illustrates one of the final stages for the parameter $a$. Bald stretches and fractal regions coexist. The particular nature of the transition region involved (reminiscent of an ecological succession) has yet to be elucidated. We conjecture that its qualitative features will constitute an invariant over large regions of parameter space.

A final observation concerns the apparent breakdown of uniqueness of the continuation of the Mandelbrot boundary away from analyticity. "Holes" appear in the interior of the bulk region (white), for $a=1.0$ close to position $c_{\mathrm{re}}=0.21$ and $c_{\text {im }}=0.76$, for example. The disappearance of the visible connection on the real axis in Fig. 6 points in the same direction.

To conclude, the Mandelbrot set apparently represents an "organizing center", in the sense of Thom [8], in the parameter space of all dynamical systems with the complexity of 2-D non-invertible maps and 3-D invertible maps [9]. The beauty of this center has yet to be unfolded.

\section{Acknowledgments}

Work is supported in part by the Stiftung Volkswagenwerk. We thank Rudolf P. Huebener, E. Held, and Claus Kahlert for discussions. 
[1] B. Mandelbrot, Ann. N.Y. Acad. Sci. 357, 249 (1980).

[2] G. Julia, J. Math. Pures et Appl. 4, 47 (1918).

[3] H. A. Lauwerier, Two-dimensional iterative maps, in: Chaos, ed. A. V. Holden, Manchester University Press, Manchester 1986, p. 58.

[4] A. Douady and J. H. Hubbard, C. R. Acad. Sci. Paris 294, 123 (1982).

[5] H. O. Peitgen and P. H. Richter, Morphology of Complex Boundaries, Exhibition Catalogue, Bremen 1984; Ber. Bunsenges. Phys. Chem. 89, 571 (1985); Phys. Bl.
42, 9 (1986); - H. O. Peitgen and P. H. Richter, The Beauty of Fractals, Springer, Berlin 1986.

[6] Note that the above pictures are all "saturation-type" results - a ten times larger step-number bound resulting in the same pictures.

[7] C. Kahlert, work in preparation.

[8] R. Thom, Stabilité Structurelle et Morphogénèse, Benjamin, Reading 1972.

[9] O. E. Rössler, C. Kahlert, J. Parisi, J. Peinke, and B. Röhricht, Z. Naturforsch. 41 a, 819 (1986). 